\title{
Oral Potentially Malignant Epithelial Lesions and Associated Risk in the Contralateral Mucosa
}

\author{
Chong Huat Siar ${ }^{1}$, Michelle Clare Mah ${ }^{1}$, Preveen Preet Gill ${ }^{1}$, Hitoshi Nagatsuka ${ }^{2}$, Hidetsugu Tsujigiwa ${ }^{2}$, \\ Mehmet Gunduz ${ }^{2}$, Noriyuki Nagai ${ }^{2}$ and $\mathrm{Kok}$ Han $\mathbf{N g}^{3}$. \\ ${ }^{1)}$ Department of Oral Pathology, Oral Medicine \& Periodontology, Faculty of Dentistry, University of Malaya, 50603 Kuala Lumpur, \\ Malaysia \\ ${ }^{2}$ Department of Oral Pathology, Okayama Graduate School of Medicine \& Dentistry, 2-5-1 Shikata-cho, Okayama, Japan \\ ${ }^{3)}$ Unit of Stomatology, Cancer Research Centre, Institute for Medical Research, Jalan Pahang, 50588 Kuala Lumpur, Malaysia
}

\begin{abstract}
Statement of the problem: Early detection of potentially malignant epithelial lesions (PMELs) is aimed at improving survival rates as carcinogenesis is a multistep process and prevention is possible if these lesions are detected at an early and reversible stage of the disease. Objectives: This prospective clinical study was designed to determine the prevalence of bilateral mirror-image PMELs in patients presenting with unilateral PMELs clinically. A modified brush biopsy technique was employed to detect early cytological epithelial changes if any, in the contralateral normal oral mucosa. Materials and method: Sixty individuals presenting with unilateral PMELs were selected for this study. These comprised 30 (50\%) Indians, 24 (40\%) Chinese, 5 (8.3\%) Malays and one (1.7\%) Nepalese. All selected cases had histopathological confirmation of their primary existing lesion(s) as inclusion criteria in this study. Cases which had subsequently presented with a lesion contralateral to the existing lesion were also subjected to scalpel incisional biopsy on this second lesion. The remaining cases which presented with a unilateral PMEL at the time of this clinical study were subjected to a brush biopsy on the clinically normal looking mucosa contralateral to the existing lesion. Results: A total of 70 lesions were detected in 60 patients. The most common PMEL found was oral lichen planus. Of the 60 patients studied, 26 exhibited mirror-image lesions either metachronously (73\%) or synchronously (27\%). The remaining cases that had undergone brush biopsy on the contralateral side to the existing PMEL yielded normal histological results. Conclusions: Present findings demonstrated that patients presenting with PMELs in the upper aerodigestive tract are at greater risk of developing a second lesion most probably on the contralateral mirrorimage site. However the efficacy of brush biopsy method in detecting epithelial changes remained inconclusive due to the small sample examined.
\end{abstract}

Keywords: Oral potentially malignant epithelial lesions, oral mucosa, clinical study, screening

\section{Introduction}

Early detection of potentially malignant epithelial lesions (PMELs) is aimed at improving survival rates as carcinogenesis is a multistep process and prevention is possible if these lesions are detected at an early and reversible stage of the disease. ${ }^{1)}$

The concept of 'field cancerization' suggests that premalignant change may occur in any area of the oral mucosa when exposed to a carcinogen (namely, tobacco and alcohol ingestion). This in turn increases the risk of patients with oral cancer developing multiple primary tumours and secondary tumour recurrence following complete excision of the primary tumour. ${ }^{2)}$

A recent study has shown that abnormal histological changes could occur in clinically normal looking mucosa of patients with oral cancer and precancerous lesions. ${ }^{3)}$

This prospective clinical study was designed to determine the prevalence of bilateral mirror-image PMELs in patients presenting with unilateral PMELs clinically.

Sample

\section{Materials and Methods}

Sixty individuals presenting with unilateral PMELs were selected

Correspondence to Chong Huat Siar,Department of Oral Pathology, Oral Medicine \& Periodontology, Faculty of Dentistry, University of Malaya, 50603 Kuala Lumpur, Malaysia, e-mail: siarch@um.edu.my, Tel: +603-7967-4859, fax:+603-7967-4531 for this study. These comprised 30 (50\%) Indians, 24 (40\%) Chinese, 5 (8.3\%) Malays and one (1.7\%) Nepalese. There were 33 (55\%) females and 27 (45\%) males, with an overall mean age of 50.2 years (Range: $30-71$ years).

\section{Histopathological examination}

All selected cases had histopathological confirmation of their primary existing lesion(s) as inclusion criteria in this study. Cases which had subsequently presented with a lesion contralateral to the existing lesion were also subjected to scalpel incisional biopsy on this second lesion.

\section{Cytobrush examination}

The remaining cases which presented with a unilateral PMEL at the time of this clinical study were subjected to a brush biopsy on the clinically normal looking mucosa contralateral to the existing lesion (Fig. 1).

The sites of all lesions and normal mucosa were charted (Fig. 2).

\section{Results and Discussion}

A total of 70 lesions were detected in 60 patients. The most common PMEL found was oral lichen planus. Of the 60 patients studied, 26 exhibited mirror-image lesions either metachronously (73\%) or synchronously (27\%). The remaining cases that had undergone brush biopsy on the contralateral side to the existing PMEL yielded normal histological results. 
International symposium of Maxillofacial \& Oral Regenerative Biology in Okayama 2005

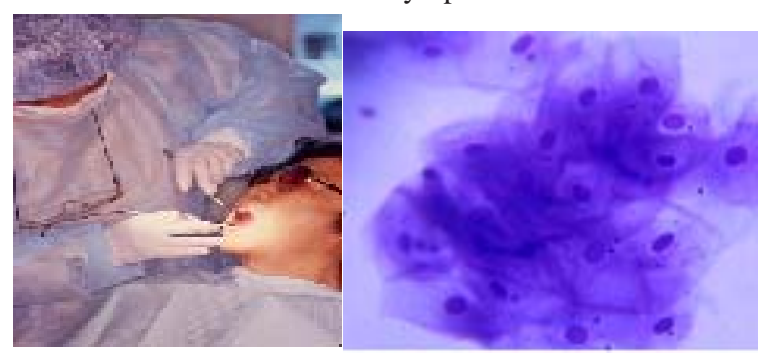

Fig. 1 Brush biopsy taking and cytosmear
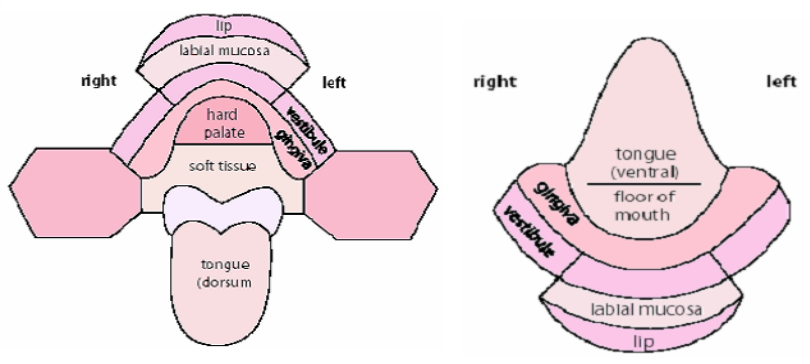

Fig. 2. Topographic chart of oral mucosa

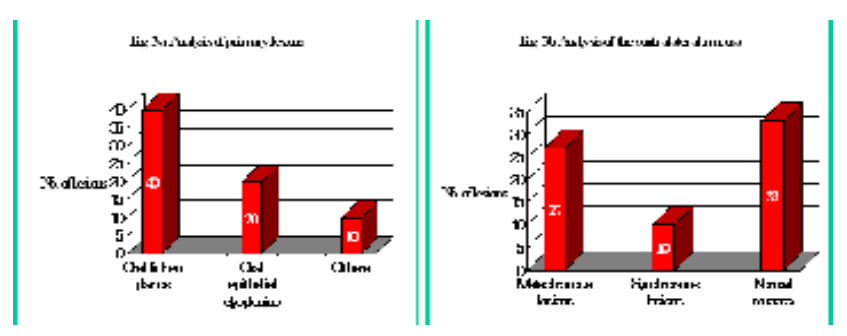

Present findings demonstrated that patients presenting with PMELs in the upper aerodigestive tract are at greater risk of developing a second lesion most probably on the contralateral mirror-image site.

However the efficacy of brush biopsy method in detecting epithelial changes remained inconclusive due to the small sample examined

\section{Acknowledgement}

We thank all staff at the Department of Oral Pathology, Oral Medicine \& Periodontology, Faculty of Dentistry, University Malaya, Unit of Stomatology, Cancer Research Centre, Institute for Medical Research, Kuala Lumpur, Malaysia, and Department of Oral Pathology, Okayama University Graduate School of Medicine \& Dentistry, Okayama, Japan for their technical assistance in this project [Ethics approval No. DF OP411/0033/ (U)].

\section{References}

1. Reibel J. Prognosis of oral premalignant lesions: Significance of clinical, histopathological and molecular biological characteristics. Crit Rev Oral Bio Med 2003;14: 47-62.

2. Slaughter DP, Southwick HW, Smejkal W. 'Field cancerization' in oral stratified squamous epithelium. Clinical implications of multicentric origin. Cancer 1953;5: 963-8.
3. Thomson PJ. Field change and oral cancer: new evidence for widespread carcinogenesis? Int J Oral Maxillofac Surg 2002;31: 262-6.

4. Potter TJ, Summerlin DJ, Campbell JH. Oral malignancies associated with negative transepithelial brush biopsy. J Oral Maxillofac Surg. 2003;61: 674-7.

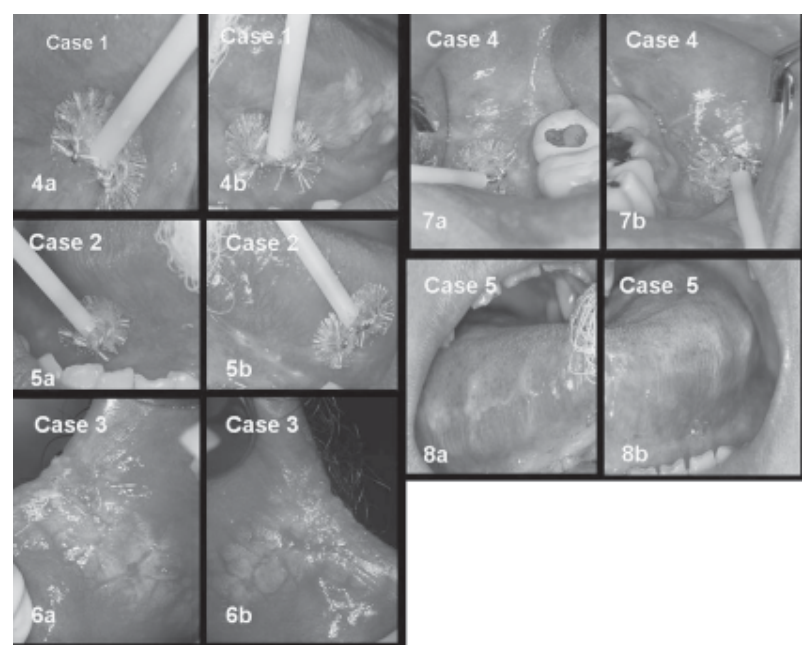

Fig. 4-8. Examples of case studied: Case 1: 47-year-old Chinese female, no habits, and presenting with leukoplakia ( $\mathrm{L}$ ) ventral tongue and normal contralateral mucosa (4a,b);Case 2: 28-year-old Nepalase male, no habits, and presenting with leukoplakia $(\mathrm{L})$ ventral tongue and normal contralateral mucosa (5a,b); Case 3: 39-year-old Indian male, smokes beedi x 25 years, and presenting with chronic hyperplastic candidiasis commissural mucosa bilaterally (6a,b); Case 4: 52-year-old Chinese female, no habits, and presenting with lichen planus/lichenoid reaction (R) and (L) buccal mucosa (7a,b); Case 5: 52-year-old Chinese female, no habits, and presenting with leukoplakia $(\mathrm{R})$ and $(\mathrm{L})$ ventrolateral tongue (8a,b). 